\title{
LA ASIGNATURA DE CURRÍCULO EN LAS FACULTADES DE CIENCIAS DE LA EDUCACIÓN: APORTES AL CAMPO DEL CURRÍCULO
}

\author{
María Elena Ortiz Espinoza*
}

\section{Resumen}

La investigación buscó conocer a través del análisis de los contenidos de la materia de Currículo y Planificación Curricular cuánto se ha avanzado en la concepción del papel de la docencia y también sobre el proceso de la formación en ésta. El estudio se restringió a tres universidades -Universidad Central del Ecuador, Universidad Técnica Particular de Loja y Universidad Politécnica Salesiana- y al currículo escrito de las asignaturas. De los resultados se pudo evidenciar que la materia aparece en 1994, reemplazando en algunos casos a la Didáctica, en otros, complementándola, y en otros como sinónimo. Surge dentro de un marco amplio de reformas educativas que se dieron en el país durante la década de los noventa, cuya finalidad fue fortalecer el aspecto pedagógico de la formación. Un carácter descriptivo y prescriptivo aún se mantiene en el abordaje de los contenidos, lo que no permite que se rompa definitivamente con la tradición tecnicista que dominó por muchos años la formación docente en nuestro país.

\section{Introducción}

El tema de la investigación nace en el contexto de la Maestría en Diseño Curricular de la Universidad Politécnica Salesiana y del "Grupo de estudios e investigación sobre currículo y culturas" que al interior de ésta se viene consolidando. Durante estos dos años de la maestría se ha podido evidenciar, entre la mayoría de los y las docentes que se integran como maestrantes, un desconocimiento de los nuevos direccionamientos y aportes que el currículo como campo de estudio y práctica ofrece al ejercicio de la docencia y a la educación de una forma más amplia.

Los supuestos sobre dicho desconocimiento fueron desde lo pedagógico hasta lo económico, pero conforme se indagó más sobre el asunto,

* Directora de la Maestría en Diseño Curricular de la Unidad de Posgrados de la Universidad Politécnica Salesiana, Ecuador. 
dos fueron los que daban las posibles respuestas. El primero se relaciona con el tipo de formación, ya que por muchos años primó la perspectiva tecnicista la cual contribuyó, entre otras cosas; a que las personas involucradas en el proceso educativo fueran divididas en dos grupos: los y las que piensan y diseñan -especialistasy los y las que ejecutan -docentes-; a que la atención del trabajo docente se centre más en el cómo, que en los qué o por qué; a que la mayoría de docentes den escasa importancia a la reflexión y actúen de forma acrítica en el ejercicio docente, y que las necesidades de actualización giren también alrededor del cómo dando poca o ninguna importancia a los aspectos teóricos de la profesión. El segundo supuesto tiene relación estrecha con el primero, ya que a pesar de que por muchos años la formación docente tanto en las universidades como en los colegios normales tuvo esas características, es de esperar que esa visión se hubiese superado porque todas las universidades en la década de los noventa reformaron sus propuestas curriculares y surgieron nuevas materias de formación pedagógica, entre ellas la materia de Currículo.

La decisión por estos supuestos obedeció a que la visión restringida centrada en el cómo, desde la década de los ochenta y noventa ya ha sido superada por los nuevos enfoques teóricos del Currículo y los aportes, sobre todo de la Sociología, la Sociología del Currículo, los Estudios Culturales, el Posestructuralismo y la Posmodernidad, los cuales han dado un giro y una perspectiva diferente sobre el rol que debe asumir el docente y el papel que desempeña la educación en la sociedad (Silva, 2004). Sin embargo de que en la literatura sobre Currículo y Prácticas Curriculares en otros países se sigue esa línea de reflexión, a través de los y las maestrantes se constató que en nuestro medio estos aportes aún son prácticamente desconocidos sumado a que la literatura sobre currículo está en inglés y portugués lo que limita aún más su difusión.

El análisis de la materia por estos motivos pasa a ser de singular importancia, ya que aparece en un contexto amplio de cambios educativos en el país, tiene un objeto de estudio bastante delimitado y una amplia literatura al respecto, además de que la visión estrecha de la formación docente, anterior a la década de los ochenta, ha sido superada, permitiendo un enriquecimiento muy importante al papel de los y las docentes en el cambio educativo.

Se escogió a tres universidades, el criterio principal fue que en dos de 
ellas no existía la materia y que luego de las reformas respectivas (Universidad Central 1992; Universidad Técnica Particular de Loja -UTPL1993 en algunas carreras) aparece por primera vez como asignatura en la formación de los y las docentes ${ }^{1}$. La Universidad Politécnica Salesiana -UPS- se escogió porque la Carrera de Pedagogía se abrió en 1996 y en su propuesta curricular aparecen materias relacionadas con el campo curricular y porque me desempeño como docente en ésta. Además se comparó con el nombre de las materias relacionadas con el currículo de la Pontificia Universidad Católica del Ecuador-PUCE - para evidenciar si la asignatura es parte de la formación en esta universidad ${ }^{2}$.

El análisis se restringió al currículo escrito de las asignaturas, tomando como referencia tanto los textos utilizados como bibliografía básica (Martín Molero, 1999 y VV. AA. 1999 /UTPL; VV. AA. 2003a y VV. AA. 2003b/ UPS; / Central) ${ }^{3}$, así como los planes analíticos de las materias. Esta delimitación se debió a la facilidad que brindan los documentos para la comparación y el análisis; además intencionalmente se dejó de lado el currículo en acción, el oculto y el mismo currículo explícito que en la realidad áulica conviven y que permitirían tener una mirada más profunda de la realidad estudiada. Esta limitación permite estar conscientes que los otros currículos son de vital importancia ya que contribuyen a tener una mayor comprensión de la totalidad, pero una investigación que tome en cuenta un mayor número de variables implica mayor profundización en varios aspectos y, por tanto, requiere mayor tiempo y otras entra-

1 Cabe aclarar que en las dos universidades se forman el mayor número de docentes tanto a nivel de bachillerato (Universidad Central del Ecuador) como de la educación básica (UTPL), mientras que en la UPS, en la Universidad Central y en la PUCE se forman docentes de Educación Inicial, por tanto pueden considerarse representativas en el contexto nacional.

2 En el marco de investigación no se analizó los contenidos de la materia en la PUCE, más por falta de tiempo para contactar a las o los docentes de la materia que por razones metodológicas. Lo que se tomó en cuenta fue los nombres de las materias, pero ya en el análisis respectivo por las razones indicadas no fueron parte de la investigación. Sería conveniente ampliar la muestra a esta universidad y realizar las respectivas comparaciones para tener una panorámica más amplia de cómo se asume la formación docente y el aporte que esta asignatura puede brindar.

3 Además del documento escrito sobre los planes analíticos, en esta universidad se conversó con uno de los docentes de la asignatura para conocer el enfoque de la asignatura, los temas tratados y la incidencia en la formación docente. 
das de análisis ${ }^{4}$. Sin embargo, al prescindir de estos elementos por los fines que persiguió la investigación no significa que el aporte inicial no sea valioso y permita comprender de manera más precisa la formación docente en nuestro país.

La pregunta central que guió la investigación fue:

¿El estudio de la materia cuánto ha contribuido a la teorización, investigación sobre el campo curricular y al cambio de la visión de ser docente?, o por el contrario, ¿se ha convertido en una materia instrumental que no aporta ni a la reflexión ni a la investigación en el campo curricular, ni al nuevo papel de los y las docentes?

Las preguntas secundarias fueron:

- ¿Qué tipo de demandas de formación hizo que la materia apareciese en el currículo?

- ¿Qué temas son abordados y cuál es su alcance?

- ¿Qué pasa con y en el campo curricular en el país?
La metodología utilizada fue el análisis de los contenidos de la materia a través de la comparación con el fin de encontrar semejanzas, diferencias, rupturas o continuidades, principalmente con la materia de Didáctica, y también para comprender su especificidad alcances y limitaciones, así como sus fortalezas o debilidades para la formación docente.

\section{Algunos hallazgos y eviden- cias sobre el tratamiento de la asignatura de Currículo}

1. La materia no existía hasta antes de 1994, pero prácticamente más de una década ha pasado desde su aparecimiento como asignatura de profesionalización.

2. Aparece en algunos casos reemplazando a la Didáctica (Currículo I -Universidad Central del Ecuador; Teoría Curricular -PUCE), en otros con el mismo nivel de importancia que la Didáctica y sin que ésta desaparezca (Cos-

4 Es imprescindible continuar con otra fase de la investigación, ya que si bien los aportes iniciales son muy valiosos, se debe profundizar aún más para tener una visión más amplia de la materia y de su incidencia en la formación docente. 
movisión del Currículo -UPS; y en el caso de la UTPL dentro de la Didáctica ya que se considera que son dos términos que hablan sobre el mismo objeto de estudio 5 .

3. Centra sus contenidos en dos aspectos de dos ámbitos del campo curricular:

- La teoría curricular (UPS, Universidad Central del Ecuador); $y$,

- La planificación curricular (Universidad Central del Ecuador, UTPL y UPS).

4. Se la conoce con diferentes nombres, según la institución universitaria:

- Cosmovisión del Currículo (UPS),

- Currículo I (Universidad Central del Ecuador),

- Teoría Curricular (PUCE).

5. La materia de Planificación Curricular es considerada una secuencia de la materia de Currículo, así como su complemento.

- Planificación Curricular (UPS y UTPL),
- Planificación y Desarrollo Curricular (PUCE),

- Currículo II (Universidad Central del Ecuador).

6. En cuanto a los contenidos específicos de la materia de Currículo tiene un espacio muy pequeño por lo que su profundización es mínima y sin los aportes desde la Teoría Curricular Crítica y Poscrítica:

- Una unidad (UPS),

- Un apartado de un capítulo (UTPL),

- Dos autores: Gonzalo Morales -colombiano- y Álvarez de Zayas -cubano(Universidad Central del Ecuador).

7. Dentro del espacio de la materia de Currículo, elementos del diseño curricular son los temas privilegiados, en el caso de la UPS y de la Universidad Central; mientras que en el caso de la UTPL los temas propios de la Didáctica son los que ocupan mayor tiempo y dedicación.

5 Es interesante la perspectiva que en el libro que utilizan para la materia de Didáctica de la autora Francisca Martín Molero ésta considera a la Teoría del Currículum como contribución para la Didáctica Científica y que la Didáctica y la Teoría del currículum son más bien términos que se emplean en el mismo sentido al igual que una misma palabra se la puede pronunciar en dos idiomas (Martín Molero 1999: 116). 
8. El ámbito del desarrollo curricular es reducido a un tema de mínimo tratamiento. En el caso de la UPS se considera como sinónimo de implementación, cuando en el campo curricular es uno de los procesos de este, pues además existen el producir y evaluar como procesos complementarios. En el caso de la Universidad Central y de la UTPL son inexistentes.

9. El énfasis curricular está en la planificación microcurricular, sobre todo en la elaboración de instrumentos de planificación. El tratamiento de estos temas son ámbito de la materia de Planificación Curricular (UPS y UTPL) y en la Central en la materia de Currículo II.

10. Los demás ámbitos del campo curricular6 (Shubert, 1986; Zaïs apud Martín Molero, 1999) no son mencionados en las dos materias, en ninguna de las universidades analizadas.

\section{Las reformas en las facultades de Ciencias de la Educación}

El primer aspecto que se evidencia es que la Materia aparece en el contexto de las reformas educativas de las universidades (Universidad Central del Ecuador y UTPL) 7 y creación en el caso de la UPS, y éstas, a su vez, están enmarcadas en el contexto sociocultural y político muy específico de la educación que se vivió, no sólo en el país sino en todo el mundo durante esos años. Recordemos que según la UNESCO y UNICEF entre marzo de 1990 y abril de 1991 terminó el siglo XX en el campo educativo, ya que durante estos meses se llevaron a cabo tres reuniones internacionales, e "introdujeron nuevos e importantes elementos en las formas de comprender los hechos educativos y en las estrategias para resolver sus principales

Según Shubert el campo curricular está formado por varios ámbitos: Teoría Curricular, Historia del currículo, Diseño curricular, Desarrollo curricular, Implementación curricular, Evaluación curricular, Cambios curriculares.

7 El caso de la Universidad Técnica Particular de Loja (UTPL) requiere mayores indagaciones, pues la materia si bien apareció con la especialización de Administración Educativa, al desaparecer ésta también desapareció la materia. Puede inferirse que el espacio nuevamente fue ocupado por la Didáctica en las demás especializaciones. 
problemas" (UNESCO UNICEF, 1991: 1). Además estas nuevas comprensiones estuvieron permeadas por los desafíos que se iban configurando cara al siglo XXI, sobre todo por la forma como los conocimientos se producían, distribuían y construían las nuevas formas de relación entre las naciones.

De estas tres reuniones mencionadas, sin lugar a dudas puede afirmarse que los compromisos asumidos luego de la Conferencia Mundial sobre Educación para Todos, en 1990, incidieron en las propuestas de reforma, pues a partir de ésta se comenzó a cuestionar sobre los resultados logrados en el proceso educativo y la necesidad de fortalecer y asegurar una educación para todos. En el caso ecuatoriano las instancias relacionadas con la Educación Ministerio, Universidades, Instituciones Educativas, Medios de Comunicación-, también coincidieron en la necesidad de realizar profundos cambios en la educación del País, ya que no respondía a las "nuevas demandas" de la sociedad.

La evidencia y énfasis sobre los resultados poco satisfactorios logrados por la educación, particularmente en países como el nuestro, llevó a pensar que la posibilidad de entrar en un mundo cada vez más globali- zado y con mercados cada vez más exigentes y especializados, era mínima. La presión a la educación, y por ende a los y las docentes para que respondan de forma favorable a estos requerimientos y para que se hagan responsables de los resultados que se buscaba conseguir, se hizo cada vez más evidente. Es así que conceptos y discursos de cómo debía ser la educación en el contexto general en aquellos años comenzaron a aparecer y a circular y, sin prácticamente darnos cuenta, se convirtieron en el eje vertebrador de lo que queríamos lograr en materia educativa. Por ello no es de extrañarse que las reformas de las universidades y de la misma Educación Básica estuvieron imbuidas, en esa época, en un discurso sobre los acelerados cambios científicos, tecnológicos y educativos, en la búsqueda de una educación de calidad para todos, en la consolidación en los y las docentes de una mejor formación y que una mayor autonomía en las decisiones pedagógicas se concreten al interior de las instituciones educativas.

Una de las limitaciones que ahora podemos evidenciar con relación a esos cambios, necesarios no sólo en la década de los noventa sino en la actualidad, es que lastimosamente respondieron más a la influencia y 
necesidades globales y particulares que a la misma realidad $\mathrm{local}^{8}$, pues los temas claves que se discutieron como metas a ser alcanzadas a través de la educación formal -valores, calidad, habilidades básicas y comunes, currículo mínimo- en realidad fueron parte de los informes "Nation at risk" 1983 y "Action for Excellence" 1983 y, por tanto, representaban lo que aquel país requería para su educación. En estos informes se afirmó que la educación -estadounidenseestaba en crisis, que habían realizado unilateralmente un "desarme educativo" y que era urgente una reforma educativa que apunte a tres metas básicas: 1. La continuación del aprendizaje para toda la vida; 2 . El asumir deberes y responsabilidades de la condición de ciudadanos; y, 3. El proporcionar habilidades básicas comunes. Éstos tres puntos se convirtieron en la bandera de lucha de todos los sectores involucrados en la educación de ese país (Apple, 1989b). Prácticamente diez años más tarde, cuando en la Conferencia Mundial para la Educación celebrada en Tailandia, se discutieron los mismos temas se asumió que todos los países participantes teníamos los mismos problemas y por ende debíamos buscar similares respuestas, por supuesto por la vía educativa (Gentili, 1995). Esta similitud lejos de contribuir a evidenciar los problemas particulares que cada país tiene, condujo a que tanto los recursos financieros como los mismos procesos de formación apunten hacia una meta común, que si bien es necesaria no debió asumirse sin tomar en cuenta las realidades locales.

Estas breves explicaciones permiten comprender el porque las reformas de las universidades se dieron prácticamente en el mismo periodo, y coinciden con el de la Educación Básica $y$, además, porque tienen como finalidad fundamental consolidar en los y las docentes "conocimientos más actualizados" acordes con un mundo globalizado; que

8 Puede evidenciarse que la realidad ecuatoriana y las necesidades propias de nuestra sociedad y de la educación de forma particular no fue tomada en cuenta, ya que no existió una verdadera evaluación del currículo que estuvo en vigencia casi 30 años, hubo un intento de reforma anterior a la vigente y de la cual ahora casi nadie se acuerda y porque el Documento final de la Reforma, prácticamente, se ha convertido en un listado de contenidos, destrezas y de algunas sugerencias metodológicas, así como de temas transversales. 
temas como aprendizajes continuos y desarrollo de habilidades básicas se conviertan en los objetivos más generales; y que el énfasis en los resultados de los logros académicos aún estén en plena vigencia. Similitudes como éstas, sin embargo, compartidas por la mayor parte de la educación ecuatoriana, no ocultan aspectos particulares que permitieron que cada universidad lleve adelante sus procesos de cambios y reestructuraciones en sus propuestas de formación. A seguir describimos brevemente estas particularidades.

La Reforma de la Facultad de Filosofía, Letras y Ciencias de la Educación de la Universidad Central del Ecuador también se debió, entre otros factores, a que los y las nuevas demandantes de las carreras en Educación venían de otros bachilleratos y no de Ciencias de la Educación. Según evaluaciones y diagnósticos se evidenció que desde su formación oficial en 1929 su sistema curricular casi se había mantenido sin modificaciones, ya que su diseño curricular respondía a las necesidades de formación de los y las estudiantes que habían terminado el bachillerato en Ciencias de la Educación o de docentes que estaban en ejercicio profesional y, por tanto, requerían un énfasis en las materias de especialización y no en la parte pedagógica, pues se asumía que éstas las habían aprendido en el Normal. No obstante, al modificarse esta tendencia se debía también cambiar el sistema curricular y que la parte de profesionalización se convierta en un aspecto importante y con el mismo peso que la parte de especialización, por este motivo materias relacionadas directamente con la formación docente pasan a ser parte del proceso de formación (Facultad de Filosofía Letras y Ciencias de la Educación. Documento de Reforma 1992-1996).

En la Pontificia Universidad Católica del Ecuador (PUCE), en la década de los noventa, se comenzó a evidenciar en las carreras de Ciencias de la Educación un lento proceso de decrecimiento en la matrícula, lo que obligó a repensar sobre la permanencia o no de algunas especializaciones como la Docencia Primaria y la Docencia Secundaria y el aparecimiento de nuevas especializaciones como el caso de la carrera de Educación Inicial y también la actualización de contenidos y materias que debían ser parte de la formación en esta nueva especialidad.

En la Universidad Politécnica Salesiana (UPS) el momento que se crea la Carrera de Pedagogía (1996) los datos estadísticos apuntaban a 
que un $73 \%$ de los y las docentes en ejercicio no tenían un título en docencia y el Ministerio de Educación, frente a estas cifras alarmantes, incentivó a que los y las docentes obtengan una titulación en Ciencias de la Educación. Para dar respuesta a esta demanda la UPS creó la especialidad de Pedagogía, pues al igual que en las otras universidades se asumió que los y las docentes lo que requerían era formación en el área pedagógica, así como una actualización de sus conocimientos, por ello el énfasis en materias de formación pedagógica antes que de especialización (VV. AA., 1997).

La Universidad Técnica Particular de Loja (UTPL) al ser una universidad de estudios a distancia, que venía funcionando desde hace algunos años atrás con el mismo sistema curricular, requería una actualización no sólo de la modalidad, sino también la apertura hacia otras carreras, incluida las de Ciencias de la Educación. La decisión de suprimir de la oferta académica la especialización de Pedagogía y crear la de Administración Educativa requirió repensar los contenidos de formación así como las materias que necesitaba el o la futura administrador/a, por lo que materias más de decisión que técnicas ocuparon un espacio importante en la formación.
Lastimosamente esta carrera tuvo pocos años de vigencia y en la actualidad ya no se oferta, sino las carreras para Educación Básica y para el bachillerato en prácticamente todas las áreas académicas.

A pesar de estas diferencias y realidades puntuales se evidencia que todas las reformas buscaron actualizar su oferta curricular, colocando el énfasis en la formación pedagógica y así los y las docentes adquieran conocimientos acordes a los nuevos escenarios y concepciones actualizadas sobre el ser humano $y$, en particular a como éste aprende; se conviertan en agentes de cambio en las instituciones educativas y que los resultados de la educación ecuatoriana mejoren. Lo que cabría después de 10 años, es que cada universidad se pregunte hasta qué punto se ha conseguido lo propuesto en cada documento de reforma y creación respectivos; dónde están insertos laboralmente los profesionales egresados de estas universidades; cuál es el grado de corresponsabilidad en los resultados poco alentadores de las pruebas; aprendió y si lo aprendido en las aulas universitarias les permitió realmente convertirse en agentes de cambio. Preguntas importantes que casi nunca en el país se hacen y tampoco se toman en cuenta el momento de hacer propuestas de 
reforma por el tiempo y costo que implica realizarlas.

\section{La asignatura y su contribu- ción en la formación docente}

Con relación al aparecimiento de la materia de Currículo y su contribución al cambio en la concepción del papel docente, puede evidenciarse que está estrechamente relacionado con la necesidad de fortalecer el aspecto pedagógico de la formación, aunque con precisión no pueda inferirse qué o quiénes contribuyeron para que esta materia sea parte importante de la formación y el porqué de sus diferentes nombres en lo que se refiere al tratamiento del objeto de estudio del currículo. Sin embargo, algunos motivos pueden explicar que la materia haya sido tomada en cuenta y que principalmente aparezca con temas propios, carga horaria importante y que algunos docentes se dediquen a su comprensión y reflexión.

El primero de estos motivos, es el contexto histórico del mismo campo del currículo en América Latina, ya que por los años noventa alcanzó un grado de madurez y de difusión en lo referente a que el papel del docente no sólo debía estar centrado en el proceso de enseñar y aprender sino que tenía implicaciones mucho más políticas, críticas y que el aspecto propositivo debía prevalecer (Moreira y Silva, 1994). Otro aspecto importante fue que en esos mismos años se buscaba romper el paradigma tecnicista que había dominado la educación por mucho tiempo y en especial la crítica acérrima hacia la obsesión por los objetivos que había dominado el escenario de la planificación y la evaluación educativa (Sacristán, 1988). Por último la difusión de libros sobre el tema en castellano, traducidos en algunos casos del inglés y otros de autores mexicanos, españoles y cubanos 9 .

En lo referente al papel del docente se afirmó, anteriormente, que por muchos años se asumió que debía estar centrado en el cómo y en el proceso de la enseñanza, especialmente en lo que se refiere a la utilización de métodos y técnicas y a las formas como se podría mejorar y llevar a cabo dicho proceso, por lo que

9 En los años noventa algunas universidades comenzaron cursos de posgrado a nivel de maestría para sus docentes en convenio con universidades extranjeras de Venezuela, México y Cuba. Esto permitió que, por un lado se de una actualización, y por otro circule literatura y autores/as que hasta aquella época no eran conocidos/as. 
aspectos técnicos como la planificación, la evaluación y el uso de materiales didácticos tuvieron un espacio muy importante y trascendental en dicha formación. Procesos de reflexión sobre selección, organización, distribución de contenidos por ejemplo, teorizaciones sobre la educación y el papel que ésta desempeña escasamente estuvieron presentes en los debates y agendas educativas. Por eso se explica que haya sido la Didáctica, entendida más como "arte de enseñar" que como ciencia, la materia que aportó con dichos conocimientos y que los temas giren alrededor del concepto y evolución de la Didáctica y un apartado grande se lo dedique a la descripción de los métodos y técnicas (Nérici 1985; Mattos, 1974).

Las Didácticas Especiales se convirtieron en el complemento de la formación y se dedicaban a enseñar las formas particulares de acuerdo a la especialización de la ciencia y niveles educativos, así como a la realización de las planificaciones de área y de clase ${ }^{10}$. La materia de Didáctica como la de Didácticas Especiales tuvieron un carácter más prescriptivo y descriptivo que reflexivo y su énfasis estaba finalmente en cómo elaborar Planes Anuales y Planes de Clase, así como el uso de las nuevas tecnologías.

Con relación a los contenidos que la materia de currículo asume puede afirmarse que existe un cierto avance en la concepción del papel de la docencia, aunque el tratamiento descriptivo aún es predominante, en el sentido que se da a conocer que existen fundamentos curriculares, niveles de concreción, bases curriculares, entre otros temas, pero no se coloca ningún énfasis en la participación del docente en dichas decisiones curriculares sino que parecería ser que otras instancias, como el Ministerio, son las encargadas de desarrollar el currículo, así como de diseñarlo y que temas como la evaluación del currículo, la misma investigación e incluso la innovación no son parte del ejercicio docente.

También puede afirmarse que se da un cierto sentido de especificidad a la materia, aunque en nuestra realidad al utilizar diferentes nombres (Teoría Curricular-Universiad Central del Ecuador, Cosmovisión del Currículo -UPS; y de la misma Didáctica -UTPL) parecería ser que

10 La materia de Didácticas especiales aún tienen su espacio, especialmente, en la formación docente del bachillerato en las diferentes especializaciones. 
su tratamiento no es unificado y el énfasis es hacia diferentes aspectos, en el primer caso más centrado en la teoría, mientras que en otro más general y en el último sin un tratamiento específico. Sin embargo, en la práctica, los temas abordados son similares tanto en la Universidad Central como en la UPS, se concentran en conceptos y definiciones del Currículo, brevemente o escasamente en la Teoría Curricular, en los fundamentos para el Diseño Curricular y los niveles de concreción, dejando para un segundo momento la planificación tal como se lo hacía con las Didácticas Especiales. Una limitación importante con este tratamiento es que se menciona sólo de forma descriptiva todos los temas, y no se profundiza en el papel que desempeña la reflexión teórica sobre el qué y el por qué y todas las implicaciones que ha tenido para y en la educación.

Puede apreciarse algunos avances pero el hecho de no reconocer toda la especificidad en la teorización del currículo implica que en nuestro país aún se desconoce por completo la conformación de la disciplina y su objeto de estudio así como:

"la existencia de amplia literatura; la atención a diversos objetos de estudio en el ámbito escolar; la selección, organización y distribución de contenidos en unas perspectivas; la realidad áulica; las fracturas, discontinuidades que en cada grupo escolar se generan; las distancias entre currículo pensado, el enseñado y el vivido; así sobre los aprendizajes valorativos no intencionados reclaman reconocer la existencia de una producción conceptual articulada a partir de una disciplina, en relación con ella y con la tarea de dar cuenta de ella (Barriga, 2003: 4).

Otra de las limitaciones importantes en el tratamiento de la asignatura es el desconocimiento de los todos los ámbitos del currículo -teoría, diseño, desarrollo, investigación, historia, innovación, implementación, evaluación-, que de acuerdo a la clasificación de Shubert (1986) implica todo un espectro de estudio, reflexión, ejecución e investigación, que también deberían ser tomados en cuenta para la comprensión y estudio del campo del Currículo, ya que si sólo se centra en una parte del diseño y de la ejecución de este -el aula-, se cae en un reduccionismo del mismo y del ejercicio de la docencia. También se puede notar un desconocimiento de los aportes sobre la historia de las disciplinas (Goodson, 1989), las relaciones entre currículo y poder (Apple, 1989a; VV. AA., 1994), el aporte de las teorías poscríticas (Silva, 2004), e incluso las mismas teorías críticas del currículo (Santos 
y Paraíso, 1996) y de la sociología del currículo (VV. AA., 1995), que en otros contextos son parte de la teorización y de la práctica curricular. Además, conceptos claves como selección, organización, distribución de los contenidos, así como las relaciones entre sociedad, culturas, poder, identidad, diferencia, tampoco son abordados en la disciplina, tomando en cuenta que estos aportes han contribuido a ampliar el debate y la inclusión de grupos y contenidos que por mucho tiempo parecerían haber sido marginados en los currículos nacionales.

Con relación a la materia de Planificación Curricular o Currículo II, la perspectiva técnica aún se mantiene, pues el tratamiento de los temas es descriptivo y el objetivo final es la elaboración de planes anuales y de aula. Si bien en esta asignatura se aborda el Proyecto Educativo Institucional (PEI), también queda en un nivel descriptivo, no existe ningún tema que explique las relaciones entre la selección, modelos de organización curricular o la misma evaluación del currículo, lo que podría llevar a que los y las docentes puedan asumir que las decisiones son incumbencia de expertos/as y que su papel debe centrarse únicamente en la ejecución de un currículo elaborado por otros/as.

\section{Conclusiones}

De las evidencias encontradas por medio de la investigación de la materia de Currículo y su complemento Planificación Curricular, podemos concluir que el tratamiento de la materia se ha quedado en un nivel instrumental, más descriptivo e incluso prescriptivo, como subsidio para la planificación microcurricular que a un nivel reflexivo, propositivo. Una de las causas es el desconocimiento de un campo de estudio amplio, desarrollado y específico, que hace que la formación docente mantenga muchos rezagos tecnicistas. Esto significa que en la formación docente aún se considera que el papel de los y las docentes debe restringirse a la ejecución de lo realizado por expertos/as desde fuera de la realidad institucional, local o particular y que no tienen ningún control sobre las decisiones curriculares.

Además, el hecho de no haber en los contenidos escritos ningún espacio para la reflexión sobre por qué ciertos contenidos se enseñan y no otros; por qué ciertos modelos de organización curricular se han mantenido casi sin ninguna modificación; cómo funciona la distribución desigual de los contenidos en ciertos contextos sociales; cómo las identidades se van construyendo a lo largo del proceso de escolarización e inclu- 
so como ciertas culturas son mostradas como válidas y silenciadas otras, restringe la relación que existe entre el currículo, la sociedad, las culturas y el poder y cómo puede esa relación contribuir a la búsqueda de alternativas a los problemas que al interior de las escuelas se suscitan.

Enfatizar en los procesos de formación el carácter propositivo y reflexivo de la actuación docente a través del currículo se hace imprescindible en nuestro país, así como el tratamiento de todos los ámbitos del campo curricular para que no sólo se conozcan sino que contribuyan a que el ejercicio de la docencia no se restrinja únicamente a aspectos prácticos, necesarios en nuestra profesión, sino también a aspectos teóricos, críticos y políticos de nuestro hacer docente.

\section{Bibliografia}

APPLE, M.

1989a Educação e poder, 201 p., Porto Alegre: Artes Médicas.

1989b Maestros y textos. Una economía política de las relaciones de clase y de sexo en educación, 218 p., Madrid: Ediciones Paidós.

BARRIGA, A.

2003 Currículum. Tensiones conceptuales y prácticas. Revista Electrónica de Investigación y
Educativa, 5 (2). Consultado el 10 de mayo del 2006 en: http://redie.uabc.mx/vol5no2/ contenido-diazbarriga.htlm. FACULTAD DE FILOSOFÍA, LETRAS Y CIENCIAS DE LA EDUCACIÓN. UNIVERSIDAD CENTRAL DEL ECUADOR Documento de Reforma 19921996. 52 p., Quito.

GENTILI, P.

1994 "O discurso da "qualidade" como nova retórica conservadora no campo educacional". In: Neoliberalismo, qualidade total e educação (Pablo A. A. Gentili e Tomaz Tadeu da Silva, orgs.): 113-177, Petrópolis: Vozes.

GOODSON, I.

1995 Currículo: teoría e historia, 140 p., Petrópolis: Vozes.

MARTÍN MOLERO, F.

1999 La Didáctica ante el tercer milenio, 287 p., Madrid: Síntesis.

MATTOS, L.

1974 Compendio de Didáctica General, 356 p., Buenos Aires: Kapeluz.

MOREIRA, A. y T. da SILVA

1994 "Sociologia e Teoria Crítica do Currículo: uma introdução". In: Currículo, Cultura e Sociedade (Antonio Flavio Moreira e Tomaz Tadeu da Silva, orgs.): 737, São Paulo: Cortez.

NERICI, I.

1985 Hacia una didáctica general dinámica, 607 p., Buenos Aires: Kapeluz. 
SACRISTÁN, G.

1990 Pedagogía por objetivos: obsesión por la eficiencia, 180 p., Madrid: Morata.

SANTOS, L y M. PARAISO

1996 O currículo como campo de lutas. Presença Pedagógica, 10 (32): 34-39.

SILVA, T. da

2004 Documentos de identidade; uma introdução às teorias do currículo, 156 p., Belo Horizonte: Autêntica.

SHUBERT, W.

1986 Curriculum. Perspective, Paradigm, and Possibility, 478 p., New York: Macmillan Publishing Company.

UNICEF UNESCO.

1991 Educación 2000. Hacia una nueva etapa de desarrollo educativo, 83 p., Quito: Imprenta Don Bosco.

VV.AA.

1994 O sujeito da educação. Estudos Foucaultianos. (Tomaz Tadeu da Silva, org.), 258 p., Petrópolis: Vozes.
VV.AA.

1995 Sociologia da educação. Dez anos de pesquisa. (Jean Claude Forquin, org.), 350 p., Petrópolis: Vozes.

VV.AA.

1999 Planificación curricular. Guía texto. (Miguel I. Valdiviezo Hidalgo, comp.), 286 p., Loja: Editorial Universidad Técnica

VV.AA.

1997 Documento de creación, 200 p., Universidad Politécnica Salesiana.

VV.AA

2003a Cosmovisión del Currículo. (Byron Falcón, comp.), 206 p., Quito: Universidad Politécnica Salesiana.

VV.AA.

2003b Planificación Microcurricular. (Byron Falcón, comp.), 105 p., Quito: Universidad Politécnica Salesiana. 